\title{
Blowup of Solution for a Class of Doubly Nonlinear Parabolic Systems
}

\author{
Jun Lu, ${ }^{1}$ Qingying Hu, ${ }^{2}$ and Hongwei Zhang ${ }^{2}$ \\ ${ }^{1}$ Department of Mathematics, Zhengzhou Normal University, Zhengzhou 450044, China \\ ${ }^{2}$ Department of Mathematics, Henan University of Technology, Zhengzhou 450001, China
}

Correspondence should be addressed to Hongwei Zhang; whz661@163.com

Received 5 August 2014; Accepted 3 November 2014; Published 18 November 2014

Academic Editor: Ajda Fošner

Copyright (c) 2014 Jun Lu et al. This is an open access article distributed under the Creative Commons Attribution License, which permits unrestricted use, distribution, and reproduction in any medium, provided the original work is properly cited.

An initial boundary value problem for a class of doubly parabolic equations is studied. We obtain sufficient conditions for the blowup of solutions under suitable initial data using differential inequalities.

\section{Introduction}

In this paper, we study the following initial boundary value problem of a class of reaction diffusion equations with multiple nonlinearities:

$$
\begin{gathered}
u_{t}-\Delta u_{t}-\Delta u+|u|^{k-2} u_{t}=|u|^{p-2} u, \\
u(x, t)=0, \quad x \in \partial \Omega, \\
u(x, 0)=u_{0}(x), \quad x \in \Omega,
\end{gathered}
$$

where $k>2, p>2$ are real numbers and $\Omega$ is bounded domain in $R^{n}$ with smooth boundary $\partial \Omega$ so that the divergence theorem can be applied. Here $\Delta$ denotes the Laplace operator in $\Omega$.

This type of problems not only is important from the theoretical point of view, but also arises in many physical applications and describes a great deal of models in applied science. It appears in the models of chemical reactions, heat transfer, and population dynamics (see [1] and references therein). Equation (1) can describe an electric breakdown in crystalline semiconductors with allowance for the linear dissipation of bound- and free-charge sources $[2,3]$.

In the absence of the nonlinear diffusion term $|u|^{k-2} u_{t},(1)$ reduced to the following equation

$$
u_{t}-\Delta u-\Delta u_{t}=|u|^{p-2} u \text {, }
$$

which is called pseudoparabolic equation (see [4] and the references). A related problem to (4) without term $-\Delta u_{t}$ has attracted a great deal of attention in the last two decades, and many results appeared on the existence, blowup, and asymptotic behavior of solution. It is well known that the nonlinear $|u|^{p-2} u$ reaction term drives the solution of (4) to blowup in finite time. The diffusion term is known to yield existence of global solution if the reaction term is removed from [5]. The more general equation,

$$
u_{t}-\operatorname{div}\left(|\nabla u|^{m-2} \nabla u\right)=f(u)
$$

has also attracted a great deal of people and the known results show that global existence and nonexistence depend roughly on $m$, the degree of nonlinearity in $f$, the dimension $n$, and the size of the initial data. See, in this regard, the works of Levine [6], Kalantarov and Ladyzhenskaya [7], Levine et al. [8], Messaoudi [9], Liu and Wang [10] and references therein. Pucci and Serrin [11] have discussed the stability of the following equation:

$$
\left|u_{t}\right|^{l-2} u_{t}-\operatorname{div}\left(|\nabla u|^{m-2} \nabla u\right)=f(u) .
$$

Levine et al. [8] got the global existence and nonexistence of solution for (6). Pang et al. $[12,13]$ and Berrimi and Messaoudi [14] gave the sufficient condition of blowup result for certain solutions of (6) with positive or negative initial energy. 
Equation (1) without term $-\Delta u_{t}$ can also be a special case of doubly nonlinear parabolic-type equations (or the porous medium equation) $[8,15,16]$,

$$
\beta(u)_{t}-\Delta u=|u|^{p-2} u,
$$

if we take $\beta(u)=u+|u|^{m-2} u$. The authors of $[15,16]$ took (7) as dynamical systems and studied their attractors. Levine and Sacks [17, 18] and Blanchard and Francfort [19] proved the existence of the solution.

Polat [20] established a blowup result for the solution with vanishing initial energy of the following initial boundary value problem:

$$
u_{t}-u_{x x}+|u|^{k-2} u_{t}=|u|^{p-2} u \text {. }
$$

They also gave detailed results of the necessary and sufficient blowup conditions together with blowup rate estimates for the positive solution of the problem,

$$
\left(u^{m}\right)_{t}-\Delta u=f(u)
$$

subject to various boundary conditions. Korpusov and Sveshnikov $[2,3]$ gave the local strong solution and the sufficient close-to-necessary conditions for the blowup of solutions to the problem,

$$
u_{t}-\Delta u_{t}-\Delta u+|u|^{k-2} u_{t}=u(u+\alpha)(u-\beta),
$$

with initial boundary values (2) and (3) in $R^{3}$ for $\alpha, \beta>0$ by the convex method $[6,7]$.

In this paper, we will investigate the problem (1)-(3) and there are few results of the problem to our knowledge. We will give sufficient conditions for the blowup of solutions in a finite time interval under suitable initial data using differential inequalities. An essential tool of the proof is an idea used in $[21,22]$, which was based on an auxiliary function (which is a small perturbation of the total energy), using differential inequalities and obtaining the result. It is different with the result of $[2,3]$. This paper is organized as follows. Section 2 is concerned with some notations and statement of assumptions. In Section 3, we give and prove that the result if the initial energy $E(0)$ of our solutions is negative (this means that our initial data are large enough) or the initial energy is $E(0)>0$.

\section{Preliminaries}

In this section, we will give some notations and statement of assumptions for $m, p, g$. We denote $L^{p}(\Omega)$ by $L^{p}, H_{0}^{1}(\Omega)$ by $H_{0}^{1}$, the usual Soblev space. The norm and inner of $L^{p}(\Omega)$ are denoted by $\|\cdot\|_{p}=\|\cdot\|_{L^{p}(\Omega)}$ and $(u, v)=\int_{\Omega} u(x) v(x) d x$, respectively. Particularly, $\|\cdot\|=\|\cdot\|_{L^{2}(\Omega)}$ for $p=2$.

For the numbers $k$ and $p$, we assume that

$$
\begin{gathered}
2<k<p \leq \frac{2(n-1)}{n-2}, \quad \text { if } n \geq 3, \\
2<k<p<+\infty, \quad \text { if } n=1,2 .
\end{gathered}
$$

Similar to [2], we call $u(x, t)$ a solution of problem (1)-(3) on $\Omega \times[0, T)$, if

$$
u \in C^{1}\left(0, T ; H_{0}^{1}\right), \quad|u|^{k-2} u_{t} \in L^{2}(\Omega \times[0, T)),
$$

satisfying $u(x, 0)=u_{0}(x)$ and

$$
\begin{gathered}
\int_{\Omega}\left[\nabla u(s) \nabla v(s)+\nabla u_{t}(s) \nabla v(s)+u_{t}(s) v(s)\right. \\
\left.+|u|^{k-2} u_{t} v-|u|^{p-2} u v\right] d x=0 \\
\forall v \in C\left(0, T ; H_{0}^{1}\right)
\end{gathered}
$$

Now, we introduce two functionals:

$$
\begin{gathered}
E(t)=E(u)=\frac{1}{2}\|\nabla u\|^{2}-\frac{1}{p}\|u\|_{p}^{p}, \\
E(0)=\frac{1}{2}\left\|\nabla u_{0}\right\|^{2}-\frac{1}{p}\left\|u_{0}\right\|_{p}^{p},
\end{gathered}
$$

where $u \in H_{0}^{1}$. Multiplying (1) by $u_{t}$ and integrating over $\Omega$, we have

$$
E^{\prime}(t)=-\left\|u_{t}\right\|^{2}-\left\|\nabla u_{t}\right\|^{2}-\int_{\Omega}|u|^{k-2} u_{t}^{2} d x<0,
$$

and then

$$
E(t) \leq E(0)
$$

\section{Blowup of Solution}

In this section, we will prove the main result. Our techniques of proof follow very carefully the techniques used in $[21,22]$.

Theorem 1. Suppose that the assumption about $k, p$ hold, $u_{0} \in$ $H_{0}^{1}$ and $u$ is a local solution of the system (1)-(3), and $E(0)<$ 0 is sufficient negative. Then the solution of the system (1)-(3) blows up in finite time.

Proof. We set

$$
H(t)=-E(t)
$$

By the definition of $H(t)$ and (15)

$$
H^{\prime}(t)=-E^{\prime}(t) \geq 0 .
$$

Consequently, by $E(0)<0$, we have

$$
H(0)=-E(0)>0 .
$$

It is clear that by (18) and (19)

$$
0<H(0) \leq H(t) .
$$

By (17) and the expression of $E(t)$,

$$
H(t)-\frac{1}{p}\|u\|_{p}^{p}=-\frac{1}{2}\|\nabla u\|^{2}<0,
$$


One implies

$$
0<H(0) \leq H(t) \leq \frac{1}{p}\|u\|_{p}^{p} .
$$

Let us define the functional

$$
L(t)=H^{1-\sigma}(t)+\frac{\epsilon}{2}\|\nabla u\|^{2}+\frac{\epsilon}{2}\|u\|^{2},
$$

where $\epsilon>0$ will be fixed in the later and $0<\sigma \leq(p-k) / p$ (this can be done since $k<p$ ). By taking the time derivative of (23) and by (1), we have

$$
\begin{aligned}
L^{\prime}(t)= & (1-\sigma) H^{-\sigma}(t) H^{\prime}(t)+\epsilon \int_{\Omega} \nabla u \nabla u_{t} d x \\
& +\epsilon \int_{\Omega} u u_{t} d x \\
= & (1-\sigma) H^{-\sigma}(t) H^{\prime}(t)+2 \epsilon H(t)+2 \epsilon E(t) \\
& -\epsilon\|\nabla u\|^{2}+\epsilon\|u\|_{p}^{p}-\epsilon \int_{\Omega}|u|^{k-2} u u_{t} d x \\
\geq & (1-\sigma) H^{-\sigma}(t) H^{\prime}(t)+2 \epsilon H(t) \\
& +\epsilon\left(1-\frac{2}{p}\right)\|u\|_{p}^{p}-\epsilon \int_{\Omega}|u|^{k-2} u u_{t} d x .
\end{aligned}
$$

To estimate the last term in the right-hand side of (24), we use the following Young's inequality

$$
a b \leq \delta^{-1} a^{2}+\delta b^{2},
$$

for any $\delta>0$, we have

$$
\begin{aligned}
\int_{\Omega}|u|^{k-2} u u_{t} d x & =\int_{\Omega}|u|^{(k-2) / 2} u_{t}|u|^{(k-2) / 2} u d x \\
& \leq \delta^{-1} \int_{\Omega}|u|^{k-2} u_{t}^{2} d x+\delta \int_{\Omega}|u|^{k} d x .
\end{aligned}
$$

Therefore, we have

$$
\begin{aligned}
L^{\prime}(t) \geq & (1-\sigma) H^{-\sigma}(t) H^{\prime}(t)+2 \epsilon H(t) \\
& +\epsilon\left(1-\frac{2}{p}\right)\|u\|_{p}^{p}-\epsilon \delta\|u\|_{k}^{k} \\
& -\epsilon \delta^{-1} \int_{\Omega}|u|^{k-2} u_{t}^{2} d x .
\end{aligned}
$$

By choosing $\delta$ such that $\delta^{-1}=M H^{-\sigma}(t)$ for $M$ enough large constants to be fixed later, and by using (15), we have

$$
\begin{aligned}
L^{\prime}(t) \geq & (1-\sigma-M \epsilon) H^{-\sigma} H^{\prime}(t)+2 \epsilon H(t) \\
& +\epsilon\left(1-\frac{2}{p}\right)\|u\|_{p}^{p}-\epsilon M^{-1} H^{\sigma}(t)\|u\|_{k}^{k} .
\end{aligned}
$$

Since $p>k>2$ and by embedding theorem, taking into account (22), we obtain, for some positive constants $C_{1}$ and $\mathrm{C}_{2}$,

$$
H^{\sigma}(t)\|u\|_{k}^{k} \leq C_{1}\|u\|_{p}^{p \sigma}\|u\|_{k}^{k} \leq C_{2}\|u\|_{p}^{p \sigma+k}
$$

Since $0<k / p<1$, now applying the inequality $x^{l} \leq(x+1) \leq$ $(1+(1 / z))(x+z)$, which holds for all $x \geq 0,0 \leq l \leq 1$, $z>0$, in particular, by the choice of $\sigma$, taking $x=\|u\|_{p}^{p}$, $l=(p \sigma+k) / p, z=H(0)$, and by using (22), we have

$$
\|u\|_{p}^{p \sigma+k} \leq\left(1+\frac{1}{H(0)}\right)\left(\|u\|_{p}^{p}+H(0)\right) \leq C_{3}\|u\|_{p}^{p} .
$$

Taking into account (28) and (30), we have

$$
\begin{aligned}
L^{\prime}(t) \geq & (1-\sigma-M \epsilon) H^{-\sigma}(t) H^{\prime}(t)+\epsilon H(t) \\
& +\epsilon\left(1-\frac{2}{p}-C_{3} M^{-1}\right)\|u\|_{p}^{p} .
\end{aligned}
$$

For large $M$ such that $1-(2 / p)-C_{3} M^{-1}=C_{4}>0$, once $M$ is fixed, we pick $\epsilon$ small enough such that $1-\sigma-M \epsilon>0$, then there exist $C_{5}>0$ such that (31) become

$$
L^{\prime}(t) \geq C_{5}\left(H(t)+\|u\|_{p}^{p}\right) .
$$

Then, we have

$$
L(t) \geq L(0) \geq 0 .
$$

On the other hand, by the definition of $L(t)$ and (21), we have

$$
\begin{aligned}
L(t) & =H^{1-\sigma}(t)-\epsilon\left(H(t)-\frac{1}{p}\|u\|_{p}^{p}\right)+\frac{\epsilon}{2}\|u\|^{2} \\
& \leq(1-\epsilon) H^{1-\sigma}(t)+\frac{\epsilon}{p}\|u\|_{p}^{p}+\frac{\epsilon}{2}\|u\|^{2},
\end{aligned}
$$

where we have used the fact $H(t) \geq H^{1-\sigma}(t)$ (this can be ensured by (19), (20), $0<\sigma<1$ and $E(0)$ is sufficient negative). Now, by inequality $x^{l} \leq(1+(1 / z))(x+z)$ again by taking $x=\|u\|_{p}^{p /(1-\sigma)}, l=1-\sigma<1, z=H^{1 /(1-\sigma)}(0)$, we have

$$
\begin{aligned}
\|u\|^{p} & \leq\left(1+\frac{1}{H^{1 /(1-\sigma)}(0)}\right)\left(\|u\|_{p}^{p /(1-\sigma)}+H^{1 /(1-\sigma)}(0)\right) \\
& \leq C_{6}\|u\|_{p}^{p /(1-\sigma)} .
\end{aligned}
$$

Therefore, we get

$$
L(t) \leq(1-\epsilon) H^{1-\sigma}(t)+C_{6}\|u\|_{p}^{p /(1-\sigma)}+\frac{\epsilon}{2}\|u\|^{2} .
$$

Then, by embedding theorem since $p>2$, we have, for fixed $\epsilon$ sufficient small,

$$
L^{1 /(1-\sigma)}(t) \leq C_{7}\left[H(t)+\|u\|_{p}^{p}+\|u\|_{p}^{2 /(1-\sigma)}\right] .
$$

Now, by inequality $x^{l} \leq(1+(1 / z))(x+z)$ again by taking $x=\|u\|_{p}^{p}, l=2 / p(1-\sigma)<1$, since $\sigma<(p-k) / p<(p-$ 2)/p, $z=H(0)$, we have

$$
\begin{aligned}
\|u\|_{p}^{2 /(1-\sigma)} & =\left(\|u\|_{p}^{p}\right)^{2 / p(1-\sigma)} \leq\left(1+\frac{1}{H(0)}\right)\left(\|u\|_{p}^{p}+H(0)\right) \\
& \leq C_{8}\|u\|_{p}^{p} .
\end{aligned}
$$


From (37) and (38), we obtain

$$
L^{1 /(1-\sigma)}(t) \leq C_{9}\left[H(t)+\|u\|_{p}^{p}\right] .
$$

Combining with (32) and (39), we arrive to

$$
L^{\prime}(t) \geq C_{10} L^{1 /(1-\sigma)}(t) .
$$

Integration of (40) between 0 and $t$ gives the desired results.

In the following, we will prove that the energy will grow up as an exponential function as time goes to infinity, provided that the initial energy $E(0)>0$.

The following lemma will play an essential role in the proof of our main result, and it is similar to a Lemma used firstly by Vitillaro [23]. In order to give the result and for the sake of simplicity, we set

$$
\lambda_{1}=C_{*}^{-p /(p-2)}, \quad E_{1}=\left(\frac{1}{2}-\frac{1}{p}\right) \lambda_{1}^{2},
$$

where $C_{*}$ is the best Poincare's constant.

Lemma 2 (see [22]). Let $u$ be a solution of (1)-(3). Suppose that the assumption of $k$, $p$ hold. Assume further that $E(0)<E_{1}$ and $\left\|\nabla u_{0}\right\|>\lambda_{1}$. Then there exists a constant $\lambda_{2}>\lambda_{1}$ such that $\|\nabla u\|>\lambda_{2}$.

Theorem 3. Suppose that the assumption about $k$, $p$ hold, $u_{0} \in$ $H_{0}^{1}$ and $u$ is a local solution of the system (1)-(3), $\left\|\nabla u_{0}\right\|>\lambda_{1}$ and $E(0)<E_{1}$. Then the solution of the system (1)-(3) blows up.

Proof. We set

$$
H(t)=E_{2}-E(t),
$$

where $E_{2}$ is a constant and $E(0)<E_{2}<E_{1}$. By the definition of $H(t)$ and (15)

$$
H^{\prime}(t)=-E^{\prime}(t) \geq 0 \text {. }
$$

Consequently,

$$
H(0)=E_{2}-E(0)>0 .
$$

It is clear that by (43) and (44)

$$
0<H(0) \leq H(t) .
$$

By (42), the expression of $E(t)$, and Lemma 2

$$
\begin{aligned}
H(t) & =E_{2}-\frac{1}{2}\|\nabla u\|^{2}+\frac{1}{p}\|u\|_{p}^{p} \\
& \leq E_{1}-\frac{1}{2} \lambda_{1}^{2}+\frac{1}{p}\|u\|_{p}^{p} \\
& =-\frac{1}{p} \lambda_{1}^{2}+\frac{1}{p}\|u\|_{p}^{p}<\frac{1}{p}\|u\|_{p}^{p} .
\end{aligned}
$$

One implies

$$
0<H(0) \leq H(t) \leq \frac{1}{p}\|u\|_{p}^{p} .
$$

Then we can prove the theorem similar to the proof of Theorem 1.

\section{Conflict of Interests}

The authors declare that there is no conflict of interests regarding the publication of this paper.

\section{Acknowledgments}

This work is supported by National Natural Science Foundation of China (no. 11171311) and partially by the Natural Science Foundation of Henan Province (1323004100360).

\section{References}

[1] Z. Jiang, S. Zheng, and X. Song, "Blow-up analysis for a nonlinear diffusion equation with nonlinear boundary conditions," Applied Mathematics Letters, vol. 17, no. 2, pp. 193-199, 2004.

[2] M. O. Korpusov and A. G. Sveshnikov, "Sufficient close-tonecessary conditions for the blowup of solutions to a strongly nonlinear generalized Boussinesq equation," Computational Mathematics and Mathematical Physics, vol. 48, no. 9, pp. 15911599, 2008.

[3] A. B. Al'shin, M. O. Korpusov, and A. G. Sveshnikov, Blowup in Nonlinear Soblev Type Equation, De Gruyter, Berlin, Germany, 2011.

[4] C. Yang, Y. Cao, and S. Zheng, "Second critical exponent and life span for pseudo-parabolic equation," Journal of Differential Equations, vol. 253, no. 12, pp. 3286-3303, 2012.

[5] K. Deng and H. A. Levine, "The role of critical exponents in blow-up theorems: the sequel," Journal of Mathematical Analysis and Applications, vol. 243, no. 1, pp. 85-126, 2000.

[6] H. A. Levine, "Some nonexistence and instability theorems for solutions of formally parabolic equations of the form $P u_{t}=$ $-A u+F(u), "$ Archive for Rational Mechanics and Analysis, vol. 51, pp. 371-386, 1973.

[7] V. K. Kalantarov and O. A. Ladyzhenskaya, "The occurrence of collapse for quasilinear equations of parabolic and hyperbolic types," Journal of Soviet Mathematics, vol. 10, no. 1, pp. 53-70, 1978.

[8] H. A. Levine, S. R. Park, and J. Serrin, "Global existence and nonexistence theorems for quasilinear evolution equations of formally parabolic type," Journal of Differential Equations, vol. 142, no. 1, pp. 212-229, 1998.

[9] S. A. Messaoudi, "A note on blow up of solutions of a quasilinear heat equation with vanishing initial energy," Journal of Mathematical Analysis and Applications, vol. 273, no. 1, pp. 243-247, 2002.

[10] W. Liu and M. Wang, "Blow-up of the solution for a $p$-Laplacian equation with positive initial energy," Acta Applicandae Mathematicae, vol. 103, no. 2, pp. 141-146, 2008.

[11] P. Pucci and J. Serrin, "Asymptotic stability for nonlinear parabolic systems," in Energy Methods in Continuum Mechanics, Kluwer Academic Publishers, Dordrecht, The Netherlands, 1996.

[12] J.-S. Pang and H.-W. Zhang, "Existence and nonexistence of the global solution on the quasilinear parabolic equation," Chinese Quarterly Journal of Mathematics, vol. 22, no. 3, pp. 444-450, 2007.

[13] J. S. Pang and Q. Y. Hu, "Global nonexistence for a class of quasilinear parabolic equation with source term and positive initial energy," Journal of Henan University (Natural Science), vol. 37, no. 5, pp. 448-451, 2007 (Chinese). 
[14] S. Berrimi and S. A. Messaoudi, "A decay result for a quasilinear parabolic system," in Elliptic and Parabolic Problems, vol. 63 of Progress in Nonlinear Differential Equations and Their Applications, pp. 43-50, 2005.

[15] A. Eden, B. Michaux, and J.-M. Rakotoson, "Doubly nonlinear parabolic-type equations as dynamical systems," Journal of Dynamics and Differential Equations, vol. 3, no. 1, pp. 87-131, 1991.

[16] H. E. Ouardi and A. E. Hachimi, "Attractors for a class of doubly nonlinear parabolic systems," Electronic Journal of Qualitative Differential Equations, vol. 2006, no. 1, pp. 1-15, 2006.

[17] H. A. Levine and P. E. Sacks, "Some existence and nonexistence theorems for solutions of degenerate parabolic equations," Journal of Differential Equations, vol. 52, no. 2, pp. 135-161, 1984.

[18] P. E. Sacks, "Continuity of solutions of a singular parabolic equation," Nonlinear Analysis: Theory, Methods \& Applications, vol. 7, no. 4, pp. 387-409, 1983.

[19] D. Blanchard and G. A. Francfort, "Study of a doubly nonlinear heat equation with no growth assumptions on the parabolic term," SIAM Journal on Mathematical Analysis, vol. 19, no. 5, pp. 1032-1056, 1988.

[20] N. Polat, "Blow up of solution for a nonlinear reaction diffusion equation with multiple nonlinearities," International Journal of Science and Technology, vol. 2, no. 2, pp. 123-128, 2007.

[21] S. Gerbi and B. Said-Houari, "Local existence and exponential growth for a semilinear damped wave equation with dynamic boundary conditions," Advances in Differential Equations, vol. 13, no. 11-12, pp. 1051-1074, 2008.

[22] B. Said-Houari, "Global nonexistence of positive initial-energy solutions of a system of nonlinear wave equations with damping and source terms," Differential and Integral Equations, vol. 23, no. 1-2, pp. 79-92, 2010.

[23] E. Vitillaro, "Global nonexistence theorems for a class of evolution equations with dissipation," Archive for Rational Mechanics and Analysis, vol. 149, no. 2, pp. 155-182, 1999. 


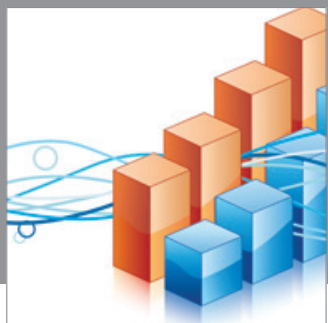

Advances in

Operations Research

mansans

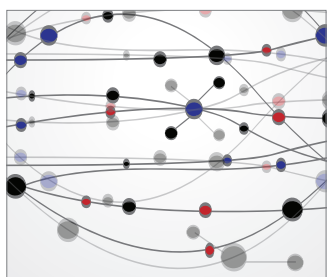

The Scientific World Journal
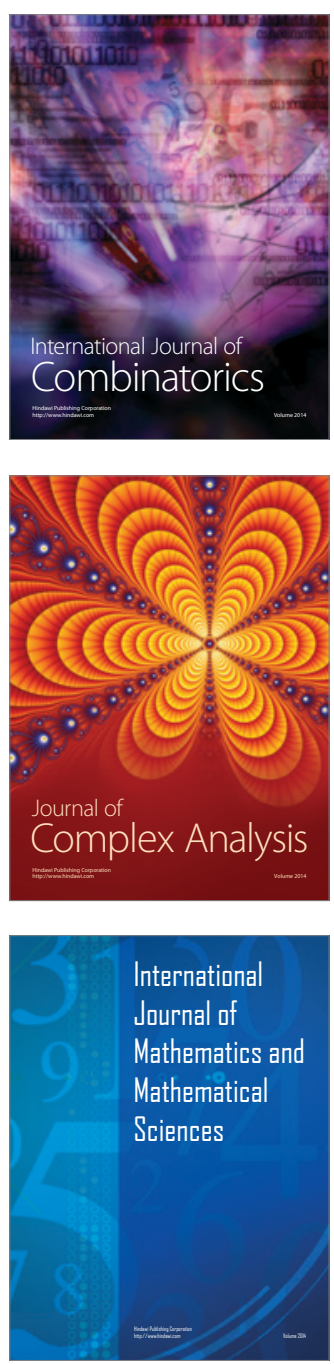
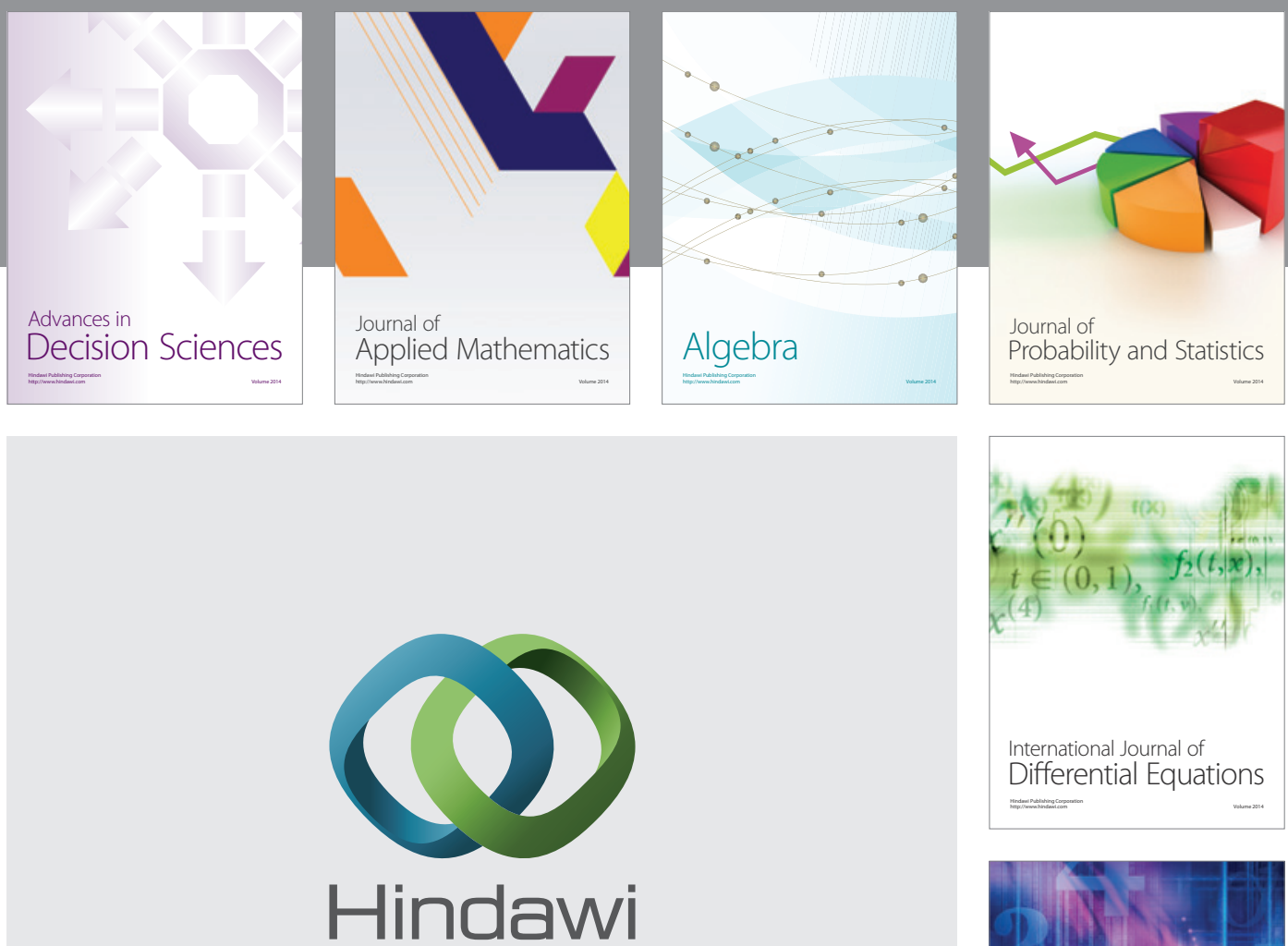

Submit your manuscripts at http://www.hindawi.com
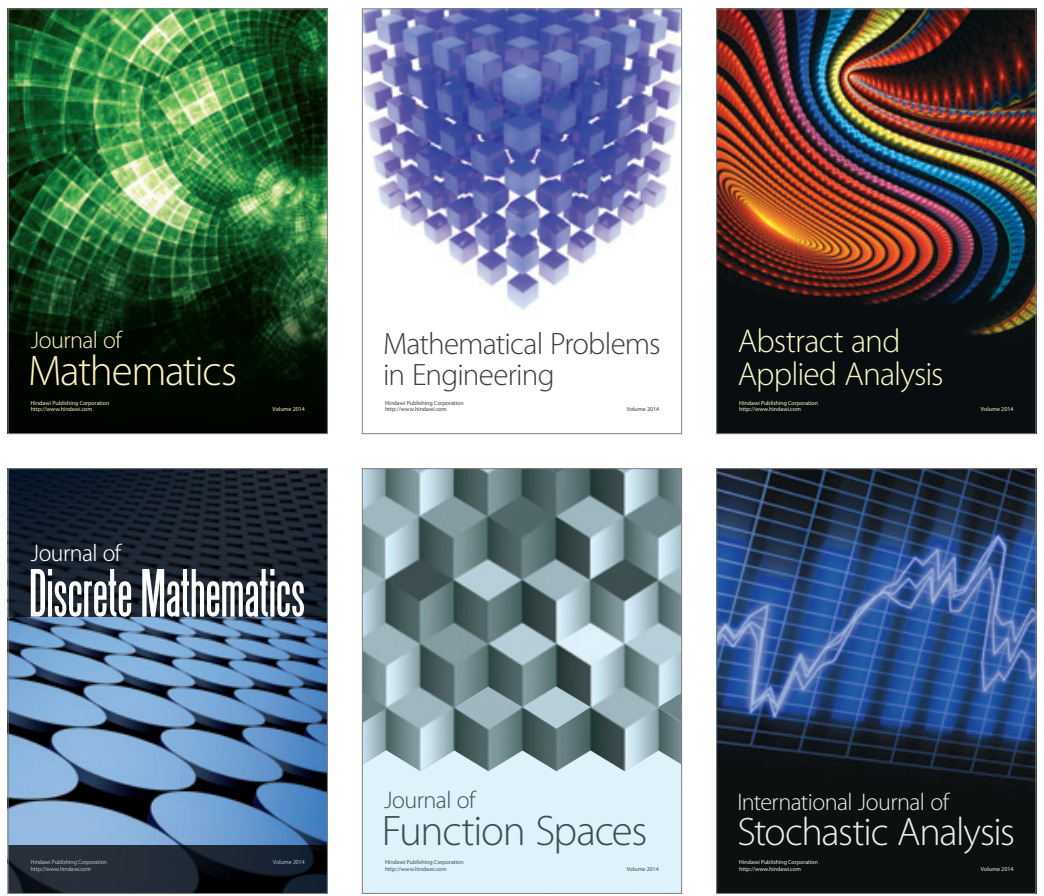

Journal of

Function Spaces

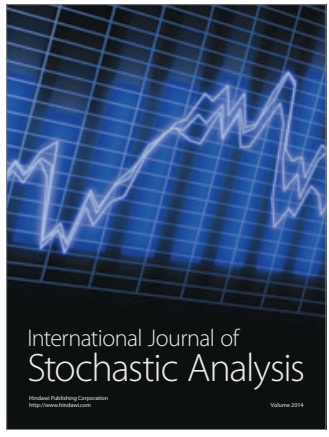

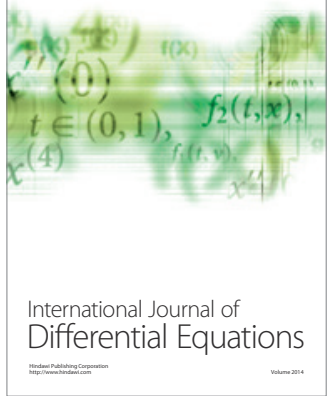
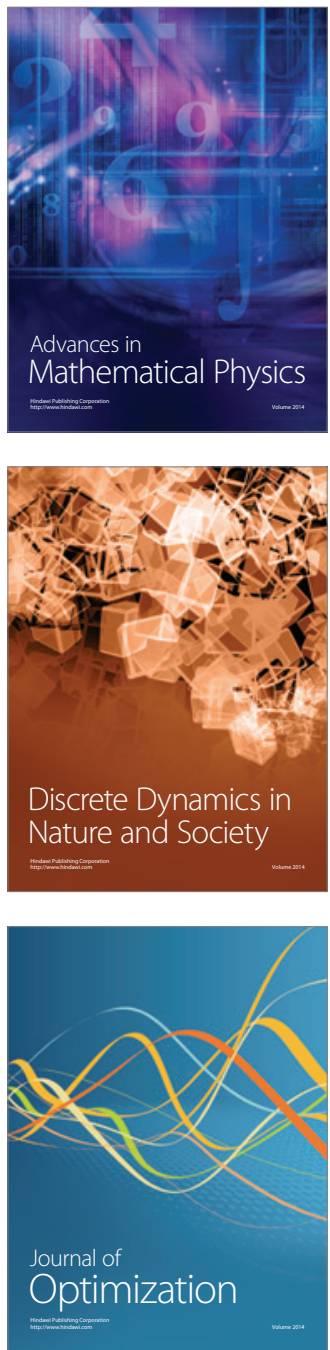